\title{
From L-shaped Planar Monopoles to a Novel Folded Antenna with Wide Bandwidth
}

\author{
Giuseppe Ruvio \\ Technological University Dublin, Giuseppe.Ruvio@tudublin.ie \\ Max Ammann \\ Technological University Dublin, max.ammann@tudublin.ie
}

Follow this and additional works at: https://arrow.tudublin.ie/engscheceart

Part of the Electrical and Computer Engineering Commons

\section{Recommended Citation}

Ruvio, G. \& Ammann, M. (2006) From L-shaped planar monopoles to a novel folded antenna with wide bandwidth. IEE Proceedings Microwaves, Antennas and Propagation, October, 2006, Vol.153, pp.456-460. doi:10.1049/ip-map:20050136

This Article is brought to you for free and open access by the School of Electrical and Electronic Engineering at ARROW@TU Dublin. It has been accepted for inclusion in Articles by an authorized administrator of ARROW@TU Dublin. For more information, please contact arrow.admin@tudublin.ie, aisling.coyne@tudublin.ie, gerard.connolly@tudublin.ie.

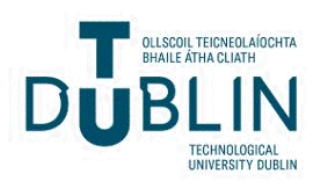




\title{
From L-shaped planar monopoles to a novel folded antenna with wide bandwidth
}

\author{
G. Ruvio and M.J. Ammann
}

\begin{abstract}
A novel folded monopole antenna is investigated numerically and experimentally. The proposed antenna comprises a short folded monopole suitably shaped at the base with two vertical grounding probes. This small antenna is shown to have a fractional impedance bandwidth up to $125 \%(1.6 \mathrm{GHz}$ to $7.5 \mathrm{GHz})$ for a $10 \mathrm{~dB}$ return loss. An interesting compromise between impedance bandwidth and pattern control is found by tuning a double side bevel at the base of the monopole, the position of the feeding point and its distance from the ground plane. Measurements and simulations are in good agreement and show a dramatic improvement in terms of impedance bandwidth compared with planar and L-shaped monopole antennas. Impedance and radiation characteristics make this novel folded monopole antenna a remarkable candidate for indoor applications where the wideband coverage of different systems is required. The working bandwidth covers a large number of wireless communication systems including GSM 1800, Personal Communication System (PCS1900), Universal Mobile Communications System (UMTS), the $2.4 \mathrm{GHz}(2400-2484 \mathrm{MHz})$ and $5.2 / 5.8 \mathrm{GHz}(5185-5350 / 5725-5875 \mathrm{MHz})$ bands for WLAN communication, Wireless Local Loop $(3.4-3.6 \mathrm{GHz})$, the US-NII bands and the lower bands allocated to ultra-wideband communications.
\end{abstract}

\section{Introduction}

Wideband antennas are becoming very attractive for future software-defined and reconfigurable wireless systems, which may operate over multiple bands. Thanks to an intrinsic broad impedance bandwidth, planar monopoles show powerful features for those applications and different feeding techniques can easily be applied to this technology to obtain further benefits [1-3]. Low-profile antennas are particularly interesting for mobile or indoor applications, so that a compromise between size and performance becomes a crucial part of the design [4-8]. Together with the introduction of special feeding techniques, reduction of the height perturbs radiation performance. Effects on impedance bandwidth and radiation pattern are examined and discussed.

Starting from a simple and fully investigated L-shaped monopole [4], the insertion of offset feeding point and bevels [2] are analysed and compared to the novel antenna proposed, in which two vertical grounding probes dramatically enlarge the impedance bandwidth with an acceptable perturbation of the radiation pattern. The horizontal plate improves radiation characteristics of the shorted monopole, whereas the shorting probes compensate for the reduction of impedance bandwidth owing to the decreased height of the antenna. Shorting elements have been already introduced in literature as a valid instrument both to control impedance bandwidth and radiation pattern [2, 5, 9].

(C) The Institution of Engineering and Technology 2006

IEE Proceedings online no. 20050136

doi:10.1049/ip-map:20050136

Paper first received 7th June and in revised form 20th September 2005

The authors are with the Centre for Telecommunications Value-chain-driven Research, School of Electronic and Communications Engineering, Faculty of Engineering, Dublin Institute of Technology, Kevin Street, Dublin 8, Ireland E-mail: max.ammann@dit.ie
Compared to typical planar monopole antennas [2, 3], this novel radiating element allows a reduction of the total height by $33 \%$. At the same time, a $10 \mathrm{~dB}$ return loss bandwidth and acceptable radiation patterns are achieved from $1.6 \mathrm{GHz}$ to $7.5 \mathrm{GHz}$.

The novel geometry proposed in this paper operates in a hybrid mode; a transmission line mode exists in addition to the monopole mode, which greatly reduces the monopole null depth and is advantageous for mobile/wireless communications. The impedance bandwidth is shown to be strongly dependent on the shape of the radiating element in proximity of the ground plane, the position of the feeding point and the feed gap. All these features are investigated and a trend of the tuning is outlined.

\section{Antennas investigated}

Figure 1 shows the antenna investigated. Starting from a simple L-shaped monopole, first a double side-bevel was introduced and analysed at the base of the radiating element. Finally, the effects of two linear probes connecting the horizontal plate to the ground plane are studied in terms of impedance bandwidth and radiation properties. All the configurations considered are vertically mounted at the centre of a $140 \times 140 \mathrm{~mm}$ ground plane, and fed by an SMA connector beneath it. The input signal is launched through a $50 \Omega$ coaxial probe of $0.6 \mathrm{~mm}$ radius with a feed gap $f g$ between the ground plane and the base of the radiating element. The antennas treated are made of $0.2 \mathrm{~mm}$-thick copper sheet.

The width $w$ is kept equal to $40 \mathrm{~mm}$ for all the cases examined throughout the paper. The same for the total height $h$, which is $f g+17 \mathrm{~mm}$ and the length of the horizontal element $l$ is equal to $24.3 \mathrm{~mm}$. The width $p$ of the shorting probes introduced in the novel monopole antenna is equal to $5 \mathrm{~mm}$. 


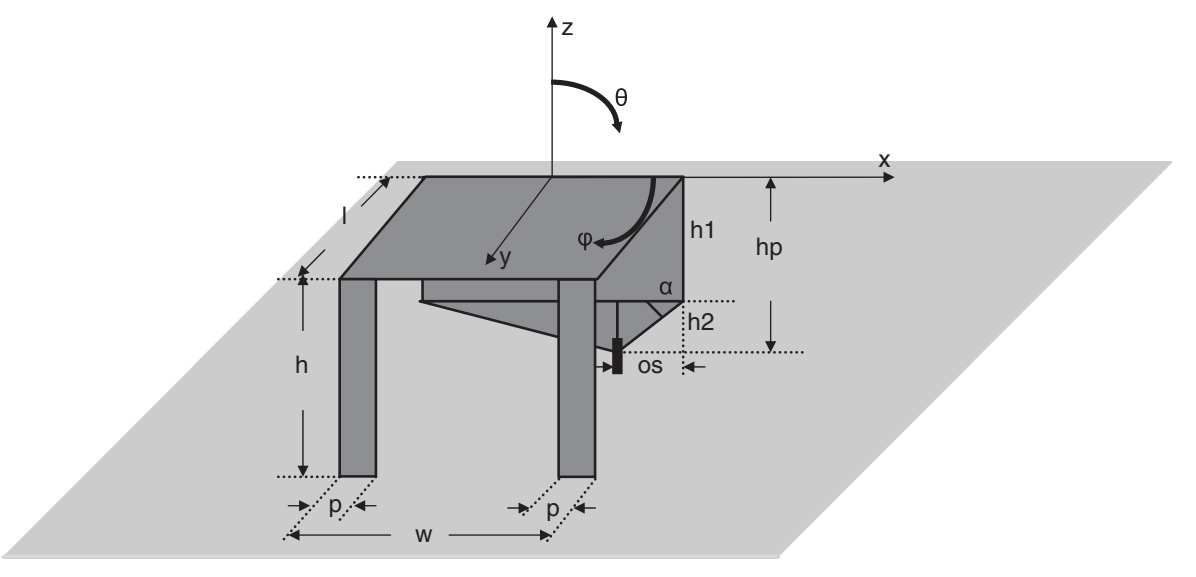

Fig. 1 Grounded L-shaped monopole

The results of bevelling the base of the L-shaped monopole are analysed in terms of four different values for $\alpha: 10^{\circ}, 15^{\circ}, 20^{\circ}$ and $25^{\circ}$. The distance os is also tuned in order to excite more modes and improve the match. Two values are considered (10 and $15 \mathrm{~mm}$ ), so that the reader can visualise the trend related to this parameter. Together with $\alpha$, os determines the partial height $h 1$ and $h 2$, in which $h_{p}$ is decomposed when the bevel is introduced.

\section{Impedance characteristics}

Initially, for the parametric analyses, the finite integration time-domain technique CST Microwave Studio is employed, and good agreement with measurement is subsequently illustrated. The first geometric parameter analysed is the feed gap, $f g$. This parameter has been demonstrated to be very critical for the matching of an L-shaped monopole [3, 10, 11]. Indeed it does affect strongly the capacitive coupling of the radiating element with the ground plane at the hottest region of the antenna. Table 1 details a comparison of simulated return loss against frequency of a simple L-shaped monopole antenna for different values of $f g$, from $0.8 \mathrm{~mm}$ to $2.3 \mathrm{~mm}$.

The widest bandwidth is obtained when $f g=1.8 \mathrm{~mm}$ and tends to decrease for larger distances from the ground plane. However, the performance changes as soon as special feeding techniques are introduced. Table 2 summarises the effect of a double side-bevel on the L-shaped monopole just studied in the previous comparison when $f g=1.8 \mathrm{~mm}$.

It appears clear that bevels dramatically extend the impedance bandwidth. A remarkable increase of the upperedge frequency is the main consequence [2]. In fact, bevelling the bottom edge of the radiating element extends the current path at the hottest part of the antenna, so that a decisive effect on the performance is expected. We obtain the best results with a double side-bevel with $\alpha=20^{\circ}$. But at the same time, a marked upward shift of the first resonance

Table 1: Effects of $f g$ on the first resonance and $10 \mathrm{~dB} R L$ impedance bandwidth with os $=0, \alpha=0$ and no shorting probes

\begin{tabular}{lll}
\hline$f g$ & Frequency range $(\mathrm{GHz})$ & Bandwidth $_{10 \mathrm{~dB}}$ \\
\hline $0.8 \mathrm{~mm}$ & $1.973-2.687$ & $30 \%$ \\
$1.3 \mathrm{~mm}$ & $2.022-2.89$ & $35 \%$ \\
$1.8 \mathrm{~mm}$ & $2.106-3.051$ & $36.6 \%$ \\
$2.3 \mathrm{~mm}$ & $2.218-3.163$ & $35 \%$ \\
\hline
\end{tabular}

is evident; from $2.645 \mathrm{GHz}$ without any bevel to $3.401 \mathrm{GHz}$ when $\alpha=20^{\circ}$. As already documented for a typical planar monopole, but also for the case of an L-shaped monopole,

Table 2: Effects of the bevel on the first resonance and $10 \mathrm{~dB} R L$ impedance bandwidth with $o s=0, f g=1.8 \mathrm{~mm}$ and no shorting probes

\begin{tabular}{lll}
\hline$\alpha$ & Frequency range $(\mathrm{GHz})$ & Bandwidth $_{10 \mathrm{~dB}}$ \\
\hline $0^{\circ}$ & $2.12-3.044$ & $35 \%$ \\
$10^{\circ}$ & $2.582-3.786$ & $37 \%$ \\
$15^{\circ}$ & $2.743-3.996$ & $37 \%$ \\
$20^{\circ}$ & $2.904-4.493$ & $43 \%$ \\
$25^{\circ}$ & $3.058-4.633$ & $40 \%$ \\
\hline
\end{tabular}

Table 3: Effects of offset on first resonance and $10 \mathrm{~dB} R L$ bandwidth with $\alpha=0, f g=1.8 \mathrm{~mm}$ and no shorting probes

\begin{tabular}{lll}
\hline os & Frequency range $(\mathrm{GHz})$ & Bandwidth $_{10 \mathrm{~dB}}$ \\
\hline- & $2.904-4.493$ & $43 \%$ \\
$10 \mathrm{~mm}$ & $2.918-8.03$ & $93 \%$ \\
$15 \mathrm{~mm}$ & $3-3.982$ & $28 \%$ \\
\hline
\end{tabular}

Table 4: Effects of $f g$ on first resonance and $10 \mathrm{~dB} R L$ bandwidth with $o s=10 \mathrm{~mm}, \alpha=10$ and no shorting probes

\begin{tabular}{lll}
\hline$f g$ & Frequency range $(\mathrm{GHz})$ & Bandwidth $_{10 \mathrm{~dB}}$ \\
\hline $0.8 \mathrm{~mm}$ & $2.946-7.608$ & $88 \%$ \\
$1.3 \mathrm{~mm}$ & $2.974-7.559$ & $87 \%$ \\
$1.8 \mathrm{~mm}$ & $3.002-3.969$ & $27.7 \%$ \\
$2.3 \mathrm{~mm}$ & $3.037-3.807$ & $22.5 \%$ \\
\hline
\end{tabular}

Table 5: Effects of $f g$ on first resonance and $10 \mathrm{~dB} R L$ bandwidth with $0 s=10 \mathrm{~mm}, \alpha=20^{\circ}$ and shorting probes

\begin{tabular}{lll}
\hline$f g$ & Frequency range $(\mathrm{GHz})$ & Bandwidth $_{10 \mathrm{~dB}}$ \\
\hline $0.8 \mathrm{~mm}$ & $1.763-7.608$ & $124 \%$ \\
$1.3 \mathrm{~mm}$ & $1.714-7.7615$ & $126 \%$ \\
$1.8 \mathrm{~mm}$ & $1.679-7.524$ & $127 \%$ \\
$2.3 \mathrm{~mm}$ & $1.644-7.349$ & $126 \%$ \\
\hline
\end{tabular}




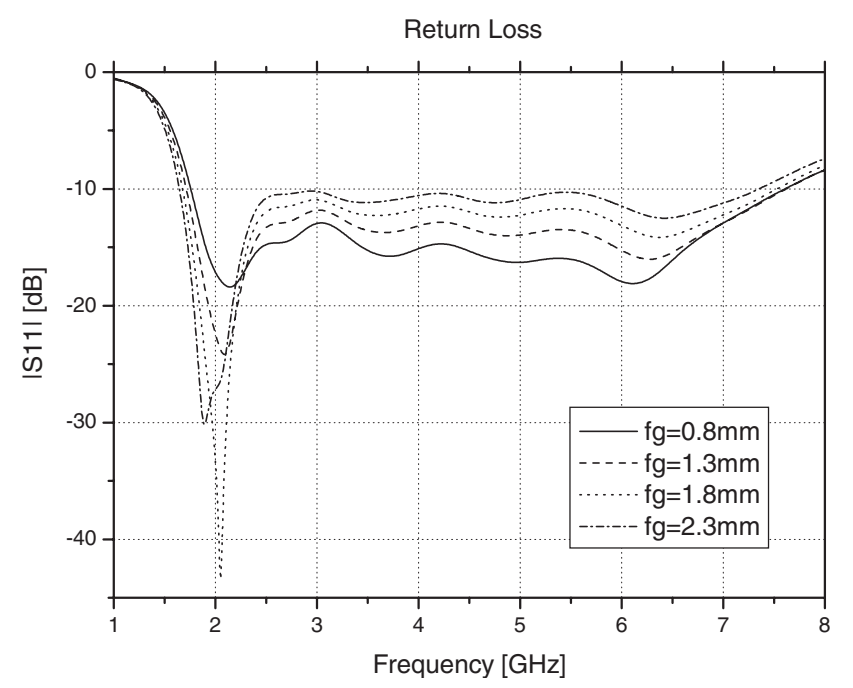

Fig. 2 Effect of $f g$ on the return loss of the L-shaped grounded monopole when $\alpha=20^{\circ}$ and $o s=10 \mathrm{~mm}$

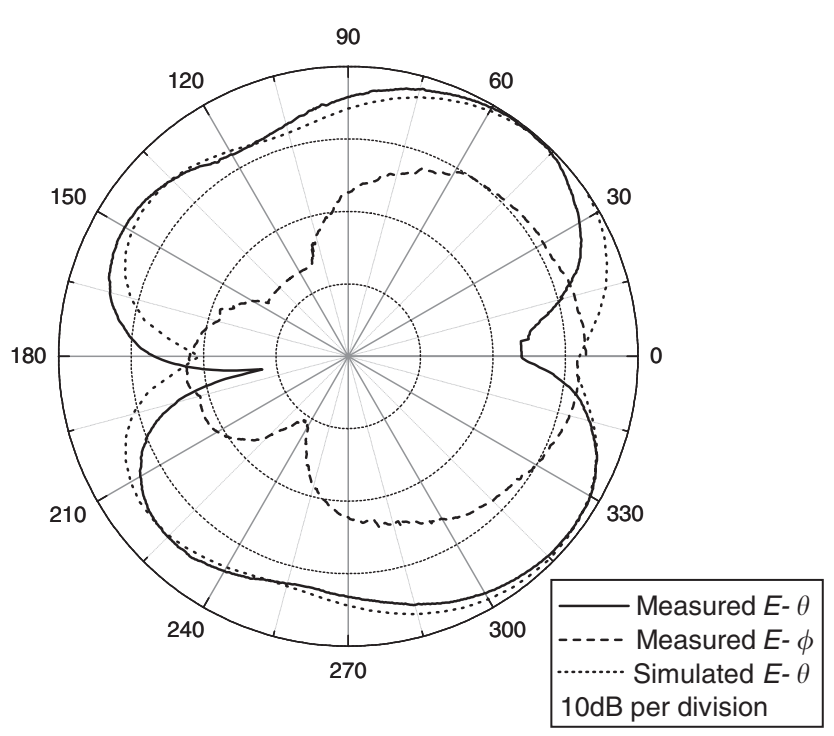

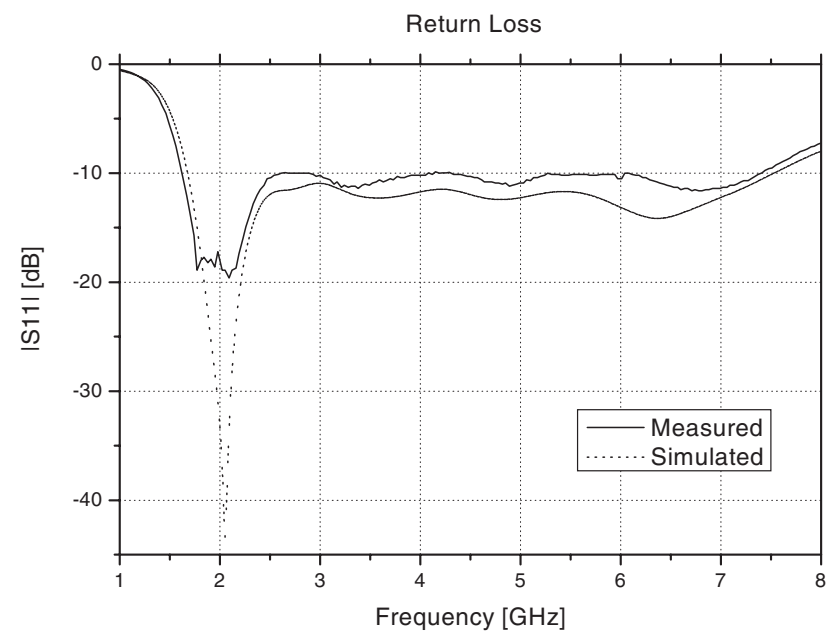

Fig. 3 Comparison of RL for the L-shaped grounded monopole in measurement and simulation
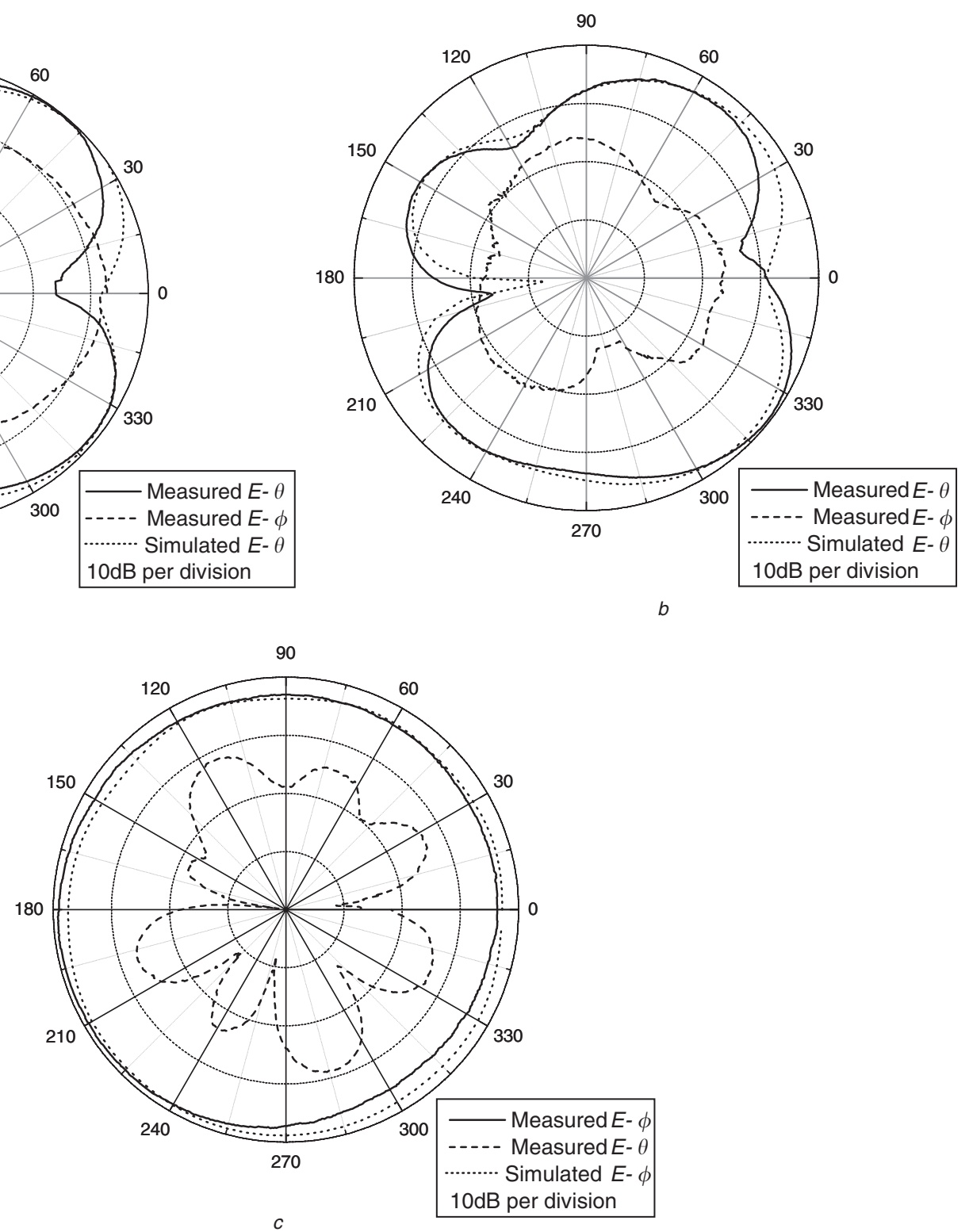

Fig. 4 Comparison of measurement and simulations of the radiation pattern of the L-shaped grounded monopole $a \alpha=20^{\circ}, f g=1.8 \mathrm{~mm}$ and $o s=10 \mathrm{~mm}$. E-plane $\varphi=0^{\circ} 1.8 \mathrm{GHz}$ $b \alpha=20^{\circ}, f g=1.8 \mathrm{~mm}$ and $o s=10 \mathrm{~mm}$. E-plane $\varphi=90^{\circ} 1.8 \mathrm{GHz}$ $c \alpha=20^{\circ}, f g=1.8 \mathrm{~mm}$ and $o s=10 \mathrm{~mm}$. H-plane $1.8 \mathrm{GHz}$ 
an enlargement of the impedance bandwidth with increase in bevel angle can be seen. In Table 2 an increase of $6 \%$ in the $10 \mathrm{~dB}$ RL bandwidth when $\alpha$ passes from $10^{\circ}$ to $20^{\circ}$ is observed.

Table 3 shows a comparison of simulated return loss when the double side bevel is kept fixed $\left(\alpha=20^{\circ}\right)$ and three different positions of the feeding point are analysed ( $f g$ remains equal to $1.8 \mathrm{~mm}$ ). As expected, by tuning os a strong reaction in terms of return loss is obtained. In fact, this geometric parameter controls the number of higher modes, which can be excited in the radiating element. The configuration $o s=10 \mathrm{~mm}$ allows the excitation of more modes than the other configurations compared.

Alternatively, when both a double side-bevel and an offset feeding point are introduced, the value of $f g=1.8 \mathrm{~mm}$ is not optimum any more. This behaviour is detailed in Table 4. As expected, by bevelling the bottom edge of the radiating element, the weight of $f g$ to control the return loss is drastically modified. Indeed it is necessary to reduce $f g$ in order to recreate a strong interaction between the monopole and the ground plane when a bevel has been introduced.

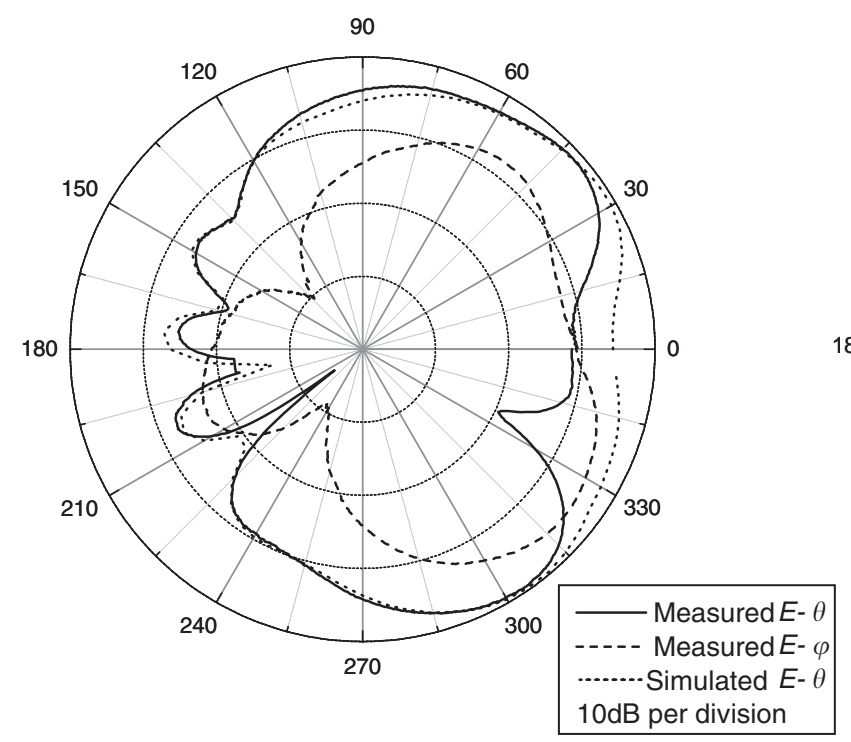

a
The best results in terms of impedance bandwidth for the L-shaped monopole with a double side-bevel $\left(\alpha=20^{\circ}\right)$ at the base and an offset of the feeding point $(f g=10 \mathrm{~mm})$ are obtained when $f g=0.8 \mathrm{~mm}$.

Finally, by introducing two shorting probes, we can notice a remarkable increase in the bandwidth. The introduction of an additional mode is the most significant consequence of this particular and novel grounding system. These vertical elements produce a significant downward shift of the lower edge frequency (see Table 5 and Fig. 2).

Feed gap, bevels, offset feed arrangements and shorting probes have been demonstrated to be very effective ways to increase the impedance bandwidth of a typical L-shaped monopole antenna. However, a strong interaction between the effects caused by these exists, so that prediction of the return loss response to tuning the structure becomes difficult, when all of them are employed simultaneously.

CST Microwave Optimizer is a tool that allows multiple tuning of geometric parameters in terms of performance goals expected. By using the interpolated quasi-Newton optimiser, the most favourable results in terms of $10 \mathrm{~dB}$ return loss impedance bandwidth were achieved for this

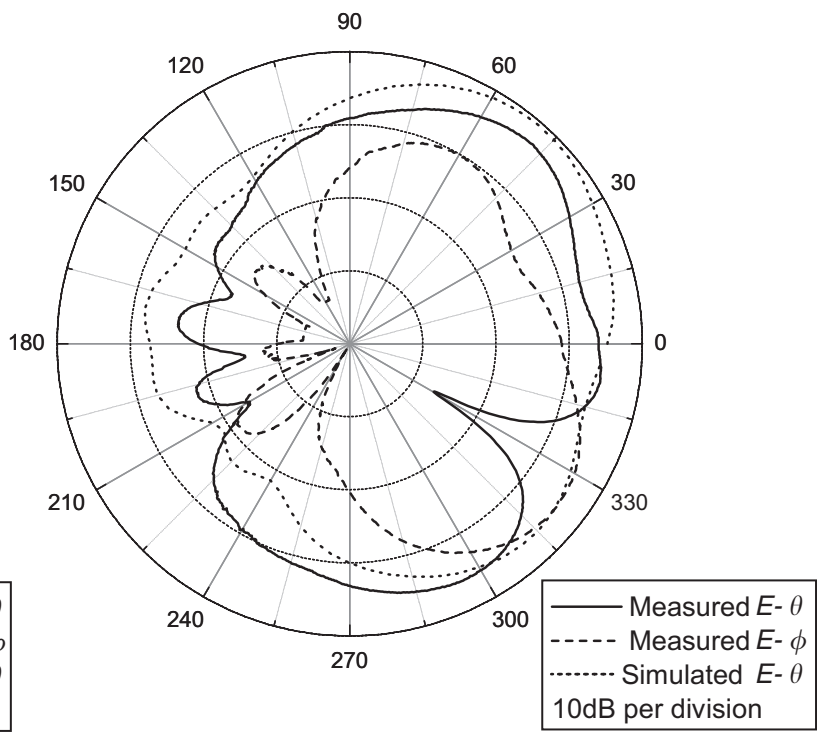

$b$

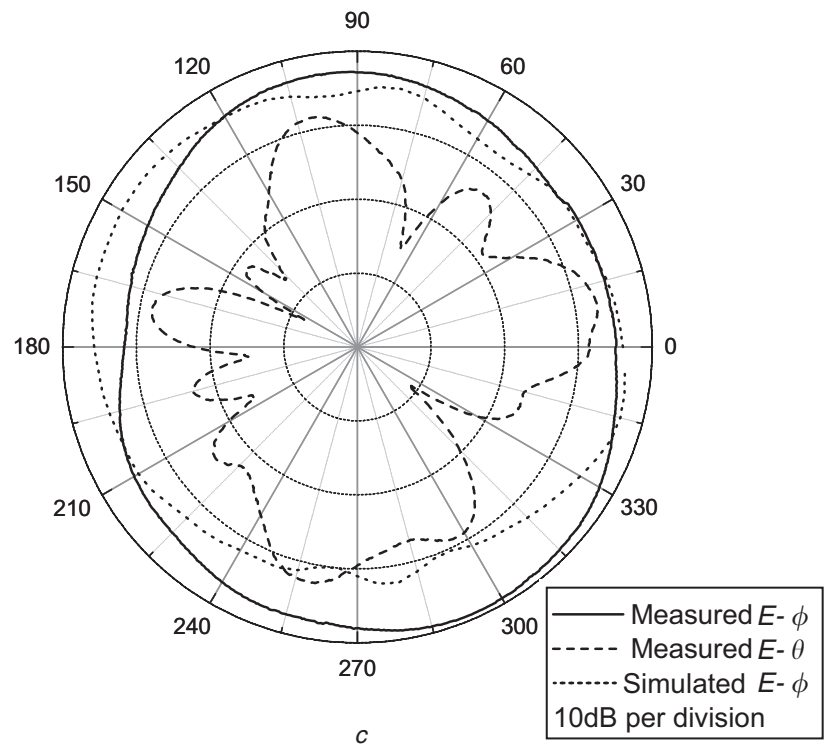

Fig. 5 Comparison of measurement and simulations of the radiation pattern of the L-shaped grounded monopole $a \alpha=20^{\circ}, f g=1.8 \mathrm{~mm}$ and $o s=10 \mathrm{~mm}$. E-plane $\varphi=0^{\circ} 4.7 \mathrm{GHz}$

$b \alpha=20^{\circ}, f g=1.8 \mathrm{~mm}$ and $o s=10 \mathrm{~mm}$. E-plane $\varphi=90^{\circ} 4.7 \mathrm{GHz}$

$c \alpha=20^{\circ}, f g=1.8 \mathrm{~mm}$ and $o s=10 \mathrm{~mm}$. H-plane $4.7 \mathrm{GHz}$ 
novel grounded L-shaped monopole antenna when $\alpha=20^{\circ}$, $o s=10 \mathrm{~mm}$ and $f g=1.8 \mathrm{~mm}$. The introduction of two symmetrical shorting probes significantly alters the current density especially at low frequency. It is believed that this new current distribution reduces the influence of $f g$ to control the return loss performance of the antenna as the capacitive coupling at the feed gap is weakened in the novel configuration. Figure 3 shows a comparison between the return loss obtained by measurement and by numerical analyses using CST Microwave Studio for the ultimate antenna obtained. A very good agreement is achieved.

\section{Radiation properties}

Measurements and simulations of the impedance properties of the antennas investigated show that the folded monopole with two grounding probes has broad impedance bandwidth, much larger than the other cases observed and with the same dimensions. Therefore, it is important to evaluate and compare the radiation properties when particular feeding techniques are introduced. Measurements and simulations are carried out in $x z, y z$ and $x y$ planes (respectively E-plane with phi $=0^{\circ}, 90^{\circ}$ and H-plane) at 1.8 and 4.7 GHz. Generally a good agreement is achieved (see Figs. $4 a-c$ and $5 a-c$ ). A gain of $4.138 \mathrm{~dB}$ and $5.71 \mathrm{~dB}$ was measured at $1.8 \mathrm{GHz}$ and $4.7 \mathrm{GHz}$ respectively. Radiation patterns in Figs. $4 a-c$ and $5 a-c$ have been normalised to the maximum gain.

It has been observed that the double side-bevel introduces a very modest perturbation on the radiation pattern of the L-shaped monopole. However, reshaping the bottom edge of the radiating element in proximity of the ground plane helps the performance in terms of omni-directionality of the H-plane pattern. This means that the bevels introduced have to be considered not only as a way to enlarge the impedance bandwidth, but also as a technique to control the pattern.

Compared to the case of a symmetric feed-point, the offset is demonstrated to have a strong effect on the pattern. The null visible at high frequencies in the $y z$ plane for $\theta \approx 45^{\circ}$ is reduced when the offset is introduced. At the same time the insertion of the offset and the shorting probes affects the typical omni-directional pattern of a monopole in the H-plane but not dramatically.

From Figs. $4 a$ and $b$ and $5 a$ and $b$, a maximum in the $x z$ and $y z$ elevation patterns is visible around $\theta=330^{\circ}$. This phenomenon is due to the presence of monopolar modes and transmission-line (patch) modes which exist, owing to the low-profile geometry [4]. This is also supported by the fact that the typical monopolar null depths are shallow and shifted from $\theta=0^{\circ}$. The two symmetrical shorting probes moderately perturb the radiation properties of a typical L-shaped antenna. This final configuration is believed to represent the best grounding solution that matches with the hybrid operating mode of this antenna. Figures $4 a-c$ and
$5 a-c$ show a comparison between measurements and simulations. A good agreement is obtained at low frequency. However, at higher frequency discrepancies become more evident.

Polarisation purity is more strongly affected at higher frequencies as shown in Fig. $5 a-c$. However, a tolerable cross-polar level is observed in the $\mathrm{H}$-plane for both of the frequencies considered. The horizontal ground plane is considered to add spurious radiation to the co-polar components of the field. This phenomenon becomes more evident as the ground plane becomes larger in terms of wavelength.

\section{Conclusions}

Effects of bevels, feed gap and asymmetric feed-point on the impedance and radiation properties of an L-shaped monopole have been carried out both experimentally and numerically. The insertion of two vertical shorting probes has been proposed and shown to be a convenient way to control impedance bandwidth and radiation pattern. Measurement data and simulations proved that vertical probes, together with bevels and offset of the feed-point, dramatically improves the performance of L-shaped planar monopoles and make them a convenient and low-cost radiation element for in-door and mobile applications where a large portion of the spectrum has to be covered.

\section{References}

1 Agrawall, N.P., Kumar, G., and Ray, K.P.: 'Wide-band planar monopole antenna', IEEE Trans. Antennas Propag., 1998, 46, (2), pp. 294-295

2 Ammann, M.J., and Chen, Z.N.: 'A wideband shorted planar monopole with bevel', IEEE Trans. Antennas Propag., 2003, 51, (4), pp. 901-903

3 Su, S.-W., Wong, K.-L., and Tang, C.-L.: 'Ultra-wideband square planar monopole antenna for IEEE 802.16a operation in the 2-11 GHz band', Microw. Opt. Technol. Lett., 2004, 42, (6), pp. $463-466$

4 Ammann, M.J., and Chen, Z.N.: 'Investigation on L-shaped planar antennas', Radio Sci., 2004, 39, p. RS2009

5 Yeh, S.-H., Chen, Y.-Y., and Wong, K.-L.: 'A low-profile, bent and shorted planar monopole antenna with reduced backward radiation for mobile phones', Microw. Opt. Technol. Lett., 2001, 33, (2), pp. $146-147$

6 Lin, S.-Y.: 'Multiband folded planar monopole antenna for mobile handset', IEEE Trans. Antennas Propag., 2004, 52, (7), pp. 1790-1794

7 Wong, K.-L., Tseng, T.-C., and Teng, P.-L.: 'Low-profile ultrawideband antenna for mobile phone applications', Microw. Opt. Technol. Lett., 2004, 43, (1), pp. 7-9

8 Huynh, M.-C., and Stutzman, W.: 'Ground plane effects on planar inverted-F antenna (PIFA) performance, IEE Proc. Microw. Antennas Propag., 2003, 150, (4), pp. 209-213

9 Tsukiji, T., Kumon, Y., and Yamasaki, M.: 'Double-folded monopole antenna using parallel line or coaxial cable', IEE Proc. Microw. Antennas Propag., 2002, 149, (1), pp. 17-22

10 Chen, Z.N., Chia, M.Y.W., and Ammann, M.J.: 'Optimisation and comparison of broadband monopoles', IEE Proc. Microw. Antennas Propag., 2003, 150, (6), pp. 429-435

11 Kumar, G., and Ray, K.P.: 'Broadband microstrip antennas' (Artech House, 2003), Chapter 9, pp. 357-378 